\title{
Credit Card FraudDetection in Payment Using Machine Learning Classifiers
}

\author{
Maad M. Mijwil ${ }^{1}$ and Israa Ezzat Salem ${ }^{2}$ \\ ${ }^{1}$ Computer Techniques Engineering Department, Baghdad College of Economic Sciences University \\ Baghdad, Iraq \\ Email: mr.maad.alnaimiy [AT] baghdadcollege.edu.iq \\ ${ }^{2}$ Computer Techniques Engineering Department, Baghdad College of Economic Sciences University \\ Baghdad, Iraq \\ Email: israa.ezzat [AT] baghdadcollege.edu.iq
}

\begin{abstract}
The fraud detection in payment is a classification problem that aims to identify fraudulent transactions based individually on the information it contains and on the basis that a fraudster's behaviour patterns differ significantly from that of the actual customer. In this context, the authors propose to implement machine learning classifiers (Nä̈ve Bayes, C4.5 decision trees, and Bagging Ensemble Learner) to predict the outco me of regular transactions and fraudulent transactions. The performance of these classifiers is $j$ judged by the follo wing ways: precision, recall rate, and precision-recall curve (PRC) area rate. The dataset includes more than $297 \mathrm{~K}$ transactions via credit cards in September 2013 and November 2017 that have been collected from Kaggle platform, of which 3293 are frauds. The performance PRC ratio of machine learning classifiers is between $99.9 \%$ and $100 \%$, which confirms that these classifiers are very goodat identifying binaryclasses 0 in the dataset. The results of the tests have proved that the bestclassifier is C4.5 decision trees. This classifier has the best accuracy of $94.12 \%$ in prediction of fraudulent transactions.
\end{abstract}

Keywords - Fraud Detection, Machine Learning, Payment, Predict, Classifiers, Credit Card.

\section{INTRODUCTION}

Card operations are carried out with credit or debit cards, which are used to buy goods and services, both in physical establishments and on the Internet [1]. Fraud in this type of operation occurs generally when the cards are copied since, in the event of theft, the customer usually notices the loss before the fraudstercan act [2]. The most common way to clone cards is to install devices in-store terminals or ATMs, which save the information of the magnetic strip when performing a regular operation. In the case of Internet purchases, fraud is even easier since knowing the card details is enough without the need for it to be present [3]. Figure 1 shows some of the cartoon images of credit card information theft (download fromGoogle).

At the present time, payment cards are one of the most popular and widely used methods by many citizens around the world. This is why so many frauds occur, and it is one of the most common problems faced by banks and payment service providers (PSP) [4]. Fraud occurs when it is not the legitimate customer who performs the operation but a third party who has managed to operate as if it were the real customer, having already saved all the bank's security mechanisms. By presenting the credit card or by announcing the credit card details (IDcard, expiration date and security code) on the Internet, it is eas y to performcard payments on the merchantside. Theresult of the low le vel of security card payment is the effect of fraudulent abuse. In addition, another major reason is the incre ase in the us e of mobile devices for payment initialization. In 2015, universal payment cards lost $\$ 21.84$ billion due to fraudulent tran sactions [6].

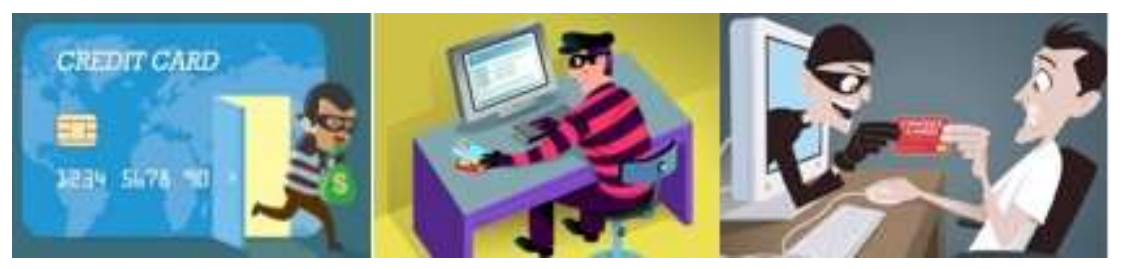

Figure 1: Cartoon images of credit card information theft. 
Forclarity, the principal contribution of our research is the application of three machine learning classifiers : (Naïve Bayes, C4.5 decision trees, and Bag ging Ensemble Learner) to detect fraud operations on $28 \mathrm{credit}$ card s. Our dat aset includes 297,467 trans actions in September 2013 and November 2017 that have been collected from the Kaggle platform, of which 3293 are frauds. This study as sists in finding the bestclas sifier out of the three classifiers used by evaluating their performance based on precision, recall rate, and precision-recall curve (PRC) area rate.

The rest of the paper is constructed as follows. Section 2, reviews some prior arts between 2017-2020. In Section 3, introduces the machine learning classifiers that will be applied in this study. In contrast, section 4 de mon strates the results obtained from these three classifiers and compares them with the results of one of the previous studies. Fin ally, conclusions are extracted in Section 5.

\section{LITERATURE SURVEY}

Many authors use machine learning classifiers to deal with the problem of card payment fraud detection. Actually, there are admittedly a large number of published papers, but in this section, we only elected six studies because they are very close to our current research. We start by Awoyemi et. al [7]. This study applies three classifiers: (Logistic Regression, K-Nearest Neighbour, and Naïve bayes) for the dataset of credit card transactions from European cardholders, containing more than $284 \mathrm{~K}$ trans actions. Theresults of this study show the greatest accuracy acc ord ing to Naïve Bayes of over $97 \%$. Yee et al. [8], dis cusses the application of machine learning classifiers (Naïve Bayes, Bayesian network clas sifiers, Tree Augmented Naïve Bayes, logistics and J48 classifiers). The results of this study achieve an accuracy of $100.0 \%$ through the J48 classifier and logistics. In the literature by Safa and Ganga [9], classifiers are implemented: Naïve Bayes, K-nearest neighbour, and Logistic Regression. The research results show th at the hig hest accuracy rate is obtained by using Logistic regression classifier, which is $97.69 \%$. In another study, Triv e di et al. [10] point out that seven machine learning classifiers are applied, such as Decision Tree, Random Forest, Naïve Bayes, Gradient Boosting, Super Vector Machine, k-Nearest Neighbour and Logistic Regression. The dat aset for th is study comprises more than $284 \mathrm{~K}$ transactions. In this study, the best accuracy is obtained by the random forest classifier exceeded $95 \%$. The study by Husejinović from BiH [11], suggests three machine learning clas sifiers (Naive Bayes, C4.5 decision tree and Bag ging Ensemble) for dataset contains more than $284 \mathrm{~K}$ trans action where 492 are fraud. The results of this study achieve more than $92 \%$ by C4.5 decision tree. This study is the closest to our current study, as its res ult s will be compared with ours. In a study conducted by Najadat et. al from Jordan [12], they discuss the application of six machine learning classifiers: Voting, Ada boosting, RandomForest, Decis ion Tree, Logistic Regression and Naïve Base. The results of this study show that the classifier with the best accuracy is the Naive bayes, which has scored over $91 \%$.

\section{THE CLASSIFIERS}

In this paper, we examine the performance of Naïve Bayes, C4.5 decision tree, and Bagging ensemble method to pass the precision, recall rate, and PRC area rate test performance. Figure 2 illustrates the mechanis m of this paper. The mechanism consists of three parts. The left side is the dataset, the middle is the applied classifiers, and the last position on the right is the outputs of these classifiers. In this section, we will briefly review each of the classifiers applied in this study. We expect it is valuable to readers.

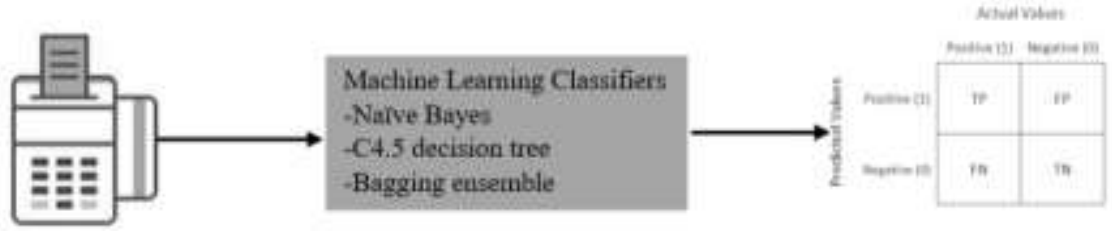

Figure 2: The mechanismof this paper [Designed by Authors].

\subsection{The first classifier is Nä̈ve Bayes}

Naïve Bayes [13][14] is a probabilistic machine learning algorithm that can be applied to a variety of clas sification tasks. This classifier is created by English mathematician Thomas Bayes. Figure 3 shows the main equation for the Naïve Bayes classifier with an explanation of each variable.

In addition, this classifier is based on Bayes' theoremand it is an idle learning model. It $\mathrm{c}$ an als o handle unstable dataset. This clas sifier works mathematically, that is, calculating the probabilities of all variables and classify ing them according to the variable with the highest probability value. On the other hand, even with little training data, the classifier can be very successful. Its advantage is that it gives a value of zero as the probability value in cas e the test data has a value that is not observed in the training data, because the result cannot be predicted. Theseconditions are commo nly referred to as zero frequency. Therefore, the correction method can be used to solve such problems, such as the application of Laplace estimation. 


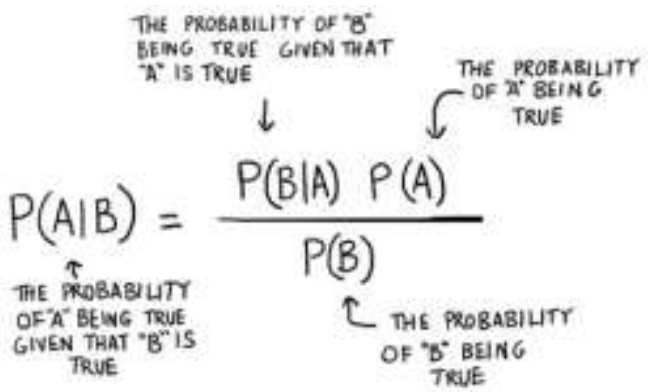

Figure 3: Naïve Bayes equation [15].

\subsection{The second classifier is C4.5 Decision Tree}

The C4.5 Decision Tree [16][17] is one of the most popular machine learning classifiers in data classification. It creates a tree structure model consis ting of decis ion nodes and terminal nodes. No matter how big the decis ion is, this clas sifier can predict the correct decision well. It is updated by dividing the data set into small parts, making it e as ier to use. The important note in this classifier is that a decision node can contain one or more branches (see Figure 4). The first node in any diagramis called a root node. Data in the decision tree can consis tof different sections, such as categ oric al and numerical data. This classifier is developed in the early 1980s by the American computer scientist J. Ros s Quinlan.

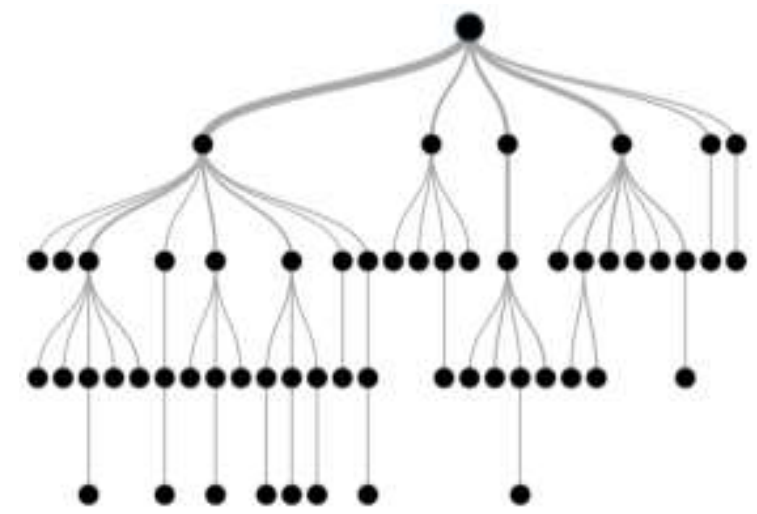

Figure 4: Example of C4.5 Decision Tree [from Google]

The dataset applied in the clas sification problemusing the decis ion tree alg orithm should be divided into two main parts (training data and testing data). The algorithmuses training datato build a model. The su ccess of the model in problem-solving is calculated by applying the model to test data.

\subsection{The third classifier is Bagging Ensemble Learning}

The Bagging Ensemble [18][19] is developed by Breiman in 1996. A collection is created by applying estimators to boot samples obtained from the original dataset. The bootloader is used here to generate refundable random selections and sub-samples. The sub-samples will be the same as the number in the original dataset. Therefore, some observations are not included in the samples created as a result of the boot, while some may be seen two or more times.

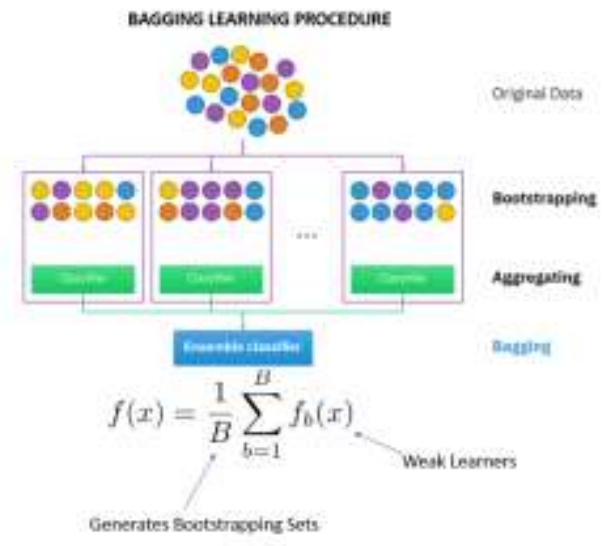

Figure 5: An example of Bagging Ens emble [20]. 
In the consolidation of estimates stage, the averages are taken for the regression trees, while the results a re determined by voting in the classification trees. Bagging can also improve the predictive effectiveness of inconsistent predictors. They can be made more convenient by using variables with small deviations but large variances. Figure 5 shows an example of Bagging Ensemble.

\section{EXPERIMENTS}

In order to verify the success of the proposed classification models, several experiments are conducted on the datase $t$ to select the best classifier. To determine the performance of the classifiers, we rely on only three outputs: Precis ion, Recall, and PRC Area. Where the higher the accuracy results, then this classifier is the best among theseexperiments. In addition, the confusion matrix provides a complete description of the performance of the clas sifier. Through this matrix, we can distinguish the correct clas sifier implementation fromthe wrong classifier implementation. Con fusion Matrix consists of two classes which are actual clas s and predicted clas s, as presented in Table 1.

Table 1: Confusion Matrix

\begin{tabular}{|c|c|c|c|}
\hline \multicolumn{4}{|c|}{ Predicted class } \\
\hline \multirow{3}{*}{$\begin{array}{l}\frac{\pi}{3} \\
\frac{D}{0} \\
\frac{0}{4}\end{array}$} & & 0 & 1 \\
\hline & 0 & True positive (TP) & False negative (FN) \\
\hline & 1 & False positive (FP) & True negative (TN) \\
\hline
\end{tabular}

Now, we calculate the Precision and Recall by formula (1) and (2), respectively:

Precision is a ratio of true positives $(T P)$ and actual positives $(T P+F P)$, by formula (1)

$$
\text { Precision }=\frac{T P}{T P+F P}
$$

Recall is a ratio of true positives $(T P)$ and actual positives $(T P+F N)$. Measures the fraction of actual positives that are correctly recognized so, by formula (2)

$$
\text { Recall }=\frac{T P}{T P+F N}
$$

The area under thePR curve is applied to meas ure the overall ability of the test to identify binary classes. It is a b asic toolfor judging models of unbalanced datasets with binary classes. The graphical vis ualization of the PRC is d es $\mathrm{cribed}$ by the recall rate on the $\mathrm{X}$-axis and the precision on the Y-axis. The higher the number, the better the performance of the clas sifier is. The original dataset is unbalanced because only $0.19 \%$ of the data is clas sified as fraud. If we predict that all data inputs will be clas sified as class 1, then we will gain $99.81 \%$ accuracy. This work is performed using Weka software v3.6 with the latest update in October 2020 with a computer: CPU: Intel@ 4 Cores-2.40GHz-Core i5-9300H, Graphics Cards: AMD Radeon XR, RAM: 8GB, and running on Ubuntu 18.04.4 LTS 64-bit. Table 2 pres ents the performance results of all applied clas sifiers. These results are applied to get precision, recall and PRC rates.

Table 2: Performance Rates

\begin{tabular}{|c|c|c|c|c|c|c|}
\hline \multirow{2}{*}{ Classifiers } & \multicolumn{2}{|c|}{ Precision } & \multicolumn{2}{c|}{ Recall } & \multicolumn{2}{c|}{ PRC Area } \\
\cline { 2 - 7 } & Class 0 & Class 1 & Class 0 & Class 1 & Class 0 & Class 1 \\
\hline Naïve Bayes & $99.9 \%$ & $65.6 \%$ & $96.5 \%$ & $81.2 \%$ & $100 \%$ & $81.0 \%$ \\
\hline C4.5 & $100 \%$ & $94.1 \%$ & $100 \%$ & $78.9 \%$ & $99.9 \%$ & $75.6 \%$ \\
\hline Bagging & $100 \%$ & $91.6 \%$ & $99.9 \%$ & $80.7 \%$ & $100 \%$ & $83.8 \%$ \\
\hline
\end{tabular}


From the table above, we note the following: The PRC area in class 0 is between $99.9 \%$ and $100 \%$, th is mean s that these classifiers are very well at dis tinguishing binary clas ses 0 in our dataset. While the PRCarea in clas s 1 , we fo und that the results in this column are different. The Naïve Bayes classifier is $81 \%$, the $\mathrm{C} 4.5$ classifier is $75.6 \%$, and the Bagging Ensemble classifier is $83.8 \%$. This experiment proves that the performance of the C4.5Decision Tree clas sifier is good, while the Bagging classifier is perfect, and the Naïve Bayes classifier is acceptable. This column is a key indicator becau se it tells us the prediction results of the clas sifiers, whether it is a regular or a fraudulent transaction. The precision of class 1 , in this column, means the precision of the predicted value of the negative value of clas s 1 . For all predicted fraudulent transactions, $94.12 \%$ will be perfectly predicted with the best achievement of the C4.5 Decision Tree clas sifier.

A comparis on of the current work is made with previous work conductedby Husejinović [11]. Table 3 illustrates the comparis on between these two studies. Through this table, we notice the success of the current work on the pre vious research in the accuracy of predicting fraudulent transactions. Also, the datasetemployed in our work is more than the previous study.

Table 3: Comparis on between current study and previous study

\begin{tabular}{|c|c|c|c|c|}
\hline Studies & Dataset & Fraud transactions & History & Precision \\
\hline Husejinović [11] & 284.807 & 492 & Sep. 2013 & $92.74 \%$ \\
\hline Our Work & 297,467 & 3293 & Sep.2013 \& Nov.2017 & $94.12 \%$ \\
\hline
\end{tabular}

\section{CONCLUSIONS}

In recent years, fraud transactions have become wides pread and have become one of the mostcritical problems facing banks all over the world. In this paper, three classifiers of machine learning are applied to predict regular or frau d u lent transactions. The best performance is C4.5 Decision Tree with $94.1 \%$ precision and $78.9 \%$ recall. The acceptable performance is Bagging Ensemble with $91.6 \%$ precision and $80.7 \%$ recall. As for the worst performance, it is the Naïve Bayes classifier. The results through this classifier are not convincing, as it gives a precision of $65.6 \%$ and a recall of $81 \%$. In the future, other classifiers will be used and applied to a s et of local data that will be collected fro $\mathrm{m}$ banks in Iraq.

\section{REFERENCES}

[1] Gupta S. and Johari R., "A New Framework for Credit Card Transactions involving Mutual Authentication betwe en Cardholder and Merchant," In Proceedings of International Conference on Commu nication Systems and Net work Technologies (CSNT), pp:22-26, Katra, India, 13-15 July 2011. https://doi.org/10.1109/CSNT.2011.12.

[2] Budhram T., "A New Framework for Credit Card Transactions Involving Mutual Authentication between Cardholder and Merchant, South African Crime Quarterly," vol.40, pp:31-37, June 2012. https://doi.org/10.17159/2413-3108/2012/v0i40a843

[3] Ermatita and Sutedja I., "Detection of Frauds for Debit Card Transactions at Automated Teller Machine in Indonesia Using Neural Network," IOP Conference Series Conference- Journal of Physics: Conference Serie s, Pale mb ang, Indonesia, pp:1-10, 26-27 November 2018. https://doi.org/10.1088/1742-6596/1196/1/012076

[4] DeVries P. D., "The problem of fraud in the banking industry: Are biometrics the answer?,"International Journal of Services and Standards, vol.7, no.3,pp:310-327, January 2011. https://doi.org/10.1504/IJSS.2011.045055

[5] Ogundele O., Zavarsky P., Ruhl R., and Lindskog D., "Fraud Reduction on EMV Pay ment Cards by the Implementation of Stringent Security Features," International Journal of Intelligent Computing Research, vol.3, no.3, pp:328-348, September 2012. https://doi.org/10.20533/ijicr.2042.4655.2012.0031

[6] Robertson D., "Top Card Issuers in Asia-Pacific," The Nilson Report, pp:1-12, October 2016. PDF link: https://nils onreport.com/upload/content promo/The Nilson Report 10-17-2016.pdf

[7] Awoyemi J. O., Adetunmbi A. O., and Oluwadare S. A., "Credit card fraud detection using machine learning techniques: A comparative analysis," In Proceedings of InternationalConference on Computing Net wo rking and Informatics (ICCNI), pp:1-6, Lagos, Nigeria, 29-31 October 2017. https://doi.org/10.1109/ICCNI.2017.8123782

[8] Yee O. S., Sagadevan S., and Malim N. H. A. H., "Credit Card Fraud Detection Using Machine Learning as Data Mining Technique,"Journal of Telecommunication, Electronic and Computer Engineering, Vol. 10 No.4, pp:23-27, August 2018.

[9] Safa M. U., and Ganga R. M., “Credit Card Fraud Detection Using Machine Learning,” International Journal of Research in Engineering, Science and Management, vol. 2, no.11,pp: 372-374, November 2019

[10] Trivedi N. K., Simaiya S., Lilhore U. K., and Sharma S. K., An Efficient Credit Card Fraud Detection ModelBased on MachineLearning Methods, International Journal of Adv anced Science and Technology, vol.29, no.5, pp:3414 3424, April 2020.

[11] Husejinović A., "Credit Card Fraud Detection Using Naive Bayesian And C4.5 Decision Tree Classifiers," Periodicals of Engineering and Natural Sciences, vol.8, no.1, pp:1-5, January 2020. 
https://doi.org/10.21533/pen.v\%25vi\%25i.300

[12] Najadat H., Altiti O., Aqouleh A. A., and Younes M., Credit Card Fraud Detection Based on Machine and Deep Learning, In Proceedings of International Conference on Information and Communication Systems (ICICS)-IEEE, pp:1-6, Irbid, Jordan, 7-9 April 2020. https://doi.org/10.1109/ICICS49469.2020.239524

[13] Chen S., Webb G. I., Liu L., and Ma X., “A Novel Selective Naïve Bayes Algorithm,” Knowledge-Based Systems Elsevier, Vol.192, March 2020. https://doi.org/10.1016/j.knosys.2019.105361

[14] Salmi N. and RustamZ., "Naïve Bayes Clas sifier Models for Predicting the Colon Cancer," IOP ConferenceSerie s Conference- Materials Science and Engineering, Malang, Indonesia, pp-1-9, 20-21 March 2019. https://doi.org/10.1088/1757-899X/546/5/052068.

[15] Sammit, "\#MLMuse-Naivety in Naive Bayes' Classifiers," Clairvoyant Soft. Available online: https://blog.clairvoyantsoft.com/mlmuse-naivety-in-naive-bayes-classifiers-9c7f6ba952bf

[16] Hsian B., Merbouha A., Ezzikouri H., And Erritali M., "A comparative study of decision tree ID3 and C4.5," International Journal of Advanced Computer Science and Applications SpecialIssue on Advances in Vehic u lar Ad Hoc Networking and Applications, pp:13-19, July 2014. http://dx.doi.org/10.14569/SpecialIssue.2014.040203

[17] Oujdi S., Belbachir H., and Boufares F., "C4.5 Decision Tree Algorithm for Spatial Data, Alternatives and Performances," Journal of Computing and Information Technology, vol.27, no.3, pp:23-29, May 2020. https://doi.org/10.20532/cit.2019.1004651

[18] Gaikwad D. P., and Thool R. C., "Intrusion Detection System Using Bagging Ensemble Method of Machine Learning," In Proceedings of International Conference on Computing Communication Control and A utomationIEEE, pp:1-6, Pune, India, 26-27 February 2015. http://doi.org/10.1109/ICCUBEA.2015.61

[19] Tuysuzoglu G. and Birant D., "Enhanced Bagging (eBagging): A Novel Approach for Ens emble Learning," The International Arab Journal of Information Technology, Vol. 17, No. 4, pp:515-528, July 2020, https://doi.org/10.34028/iajit/17/4/10

[20] Singhal G., "Ensemble Methods in MachineLearning: Bagging Versus Boosting," Pluralsight, June 2020. Available online: https://www.pluralsight.com/guides/ensemble-methods:-bagging-versus-boosting 